\title{
Transport Characteristics of Charge Carriers in Normal State Superconductor $\mathrm{YBa}_{2} \mathrm{Cu}_{3} \mathrm{O}_{7-\delta}$
}

\author{
Vilius Palenskis \\ Physics Faculty, Vilnius University, Vilnius, Lithuania \\ Email: vilius.palenskis@ff.vu.It
}

Received 27 May 2015; accepted 27 July 2015; published 30 July 2015

Copyright (c) 2015 by author and Scientific Research Publishing Inc. This work is licensed under the Creative Commons Attribution International License (CC BY). http://creativecommons.org/licenses/by/4.0/

\section{cC) (†) Open Access}

\begin{abstract}
The general expressions based on the Fermi distribution of the free charge carriers are applied for estimation of the transport characteristics in superconductors at the temperature well above the superconducting phase transition temperature $T_{c}$. The Hall-effect experimental results in the normal state of the superconductor $\mathrm{YBa}_{2} \mathrm{Cu}_{3} \mathrm{O}_{7-\delta}$ are not finally explained. On the ground of the randomly moving charge carriers, the transport characteristics of the randomly moving charge carriers for both single type and two types of the charge carriers are presented. The particular attention has been pointed to the Hall-effect measurement results of the high- $T_{C}$ superconductor $\mathrm{YBa}_{2} \mathrm{Cu}_{3} \mathrm{O}_{7-\delta}$. It is at the first time derived the Hall coefficient expression for two type of highly degenerate charge carriers (electrons and holes) on the ground of the randomly moving charge carriers at the Fermi surface. It is shown that the Hall coefficient and other transport characteristics are determined by the ratio between the electron-like and hole-like densities of states at the Fermi surface.
\end{abstract}

\section{Keywords}

Randomly Moving Charge Carrier Density, Electrical Conductivity, Two-Band Model, Hall Coefficient, Hall Mobility, Drift Mobility, Density of States

\section{Introduction}

In all cuprates whose doping has been optimized for maximum superconducting transition temperature $T_{C}$, the resistivity $\rho(T)$ well above $T_{C}$ is quite accurately linear in $T$ as for ordinary metals. So, the resistivity is proportional to $1 / \tau$ (here $\tau$ is the average electron scattering time). In metal the Hall coefficient is independent on temperature which is considered that mobile carrier density is constant and equal to the valence electron density. As it is presented in [1]-[3], the conductivity is determined by the effective density of randomly moving charge 
carriers $n_{\text {eff. }}$ For metals, normal state superconductors and other materials with degenerate electron gas the effective density of randomly moving charge carriers is determined by the density of states $g\left(E_{F}\right)$ at the Fermi energy $E_{F}: n_{\text {eff }}=g\left(E_{F}\right) k T$ (here $k$ is the Boltzmann constant, and $T$ is the absolute temperature). The density of states $g\left(E_{F}\right)$ is obtained from the experimental results of the electron heat capacity measurements. For multivalent metals and superconductors in the normal state with the composite density of states, the Hall coefficient does determine neither the total density of free electrons, nor the effective density of randomly moving charge carriers.

How to understand the temperature dependences of both resistivity $\rho(T)$ and Hall coefficient $R_{H}(T)$ of $\mathrm{YBa}_{2} \mathrm{Cu}_{3} \mathrm{O}_{7}$ (YBCO) in terms of a unified physical picture is still an open question. In almost all works the explanation of the resistivity $\rho_{a b}(T)$ in $a b$ plane is based upon a 2D single-band model for holes. The nearly linear $T$ dependence of $\rho_{a b}(T)$ is obtained by assuming the free density of charge carriers to be constant and deducing the approximate relation $1 / \tau \sim T$ from electron-phonon interaction. In YBCO there is also an appreciable anisotropy of conductivity within $a b$ plane, as one will expect because of the mobile holes on the chains. For YBCO optimized for maximum $T_{C}$ the resistivity $\rho_{a}$ in $a$ direction is about double of $\rho_{b}$ in $b$ direction, and they have the same temperature dependences [4]. The ratio $\rho_{a} / \rho_{b}$ value is about 2.2 and is independent on temperature; it indicates that the $\mathrm{Cu}-\mathrm{O}$ chains contribute about $60 \%$ of the current when the electric field is parallel to them, with the rest of the current being contributed by the $\mathrm{Cu}-\mathrm{O}$ planes. The electrical resistivity in the $c$ direction is high, as it is expected from the localization in the $c$ direction of the most electron states in the planes. It is believed that they are tunneling from one layer to the next in the $c$ direction [5]. On the other hand, the measurement of the Hall coefficient for a single-crystal YBCO shows that for magnetic field parallel to $c$ axis and for the direct current in $a b$ plane $R_{H}$ is proportional to $1 / T$, and the charge carriers are holes with $R_{H}>0$.

A study is usually starting from a single parabolic band picture, but there is a problem on explanation of temperature dependences of both conductivity and Hall coefficient: the former implying that $n \approx$ const with $1 / \tau \sim T$, and another implying that $n \sim T$ which follows from the Hall effect measurement. So, it shows that for a high- $T_{C}$ superconductors with complex band structure the free-electron formulas

$$
\sigma=q n \mu=q^{2} n \tau / m
$$

and

$$
R_{H}=1 / q n
$$

are not longer hold. As it is shown in [1]-[3], these formulas are valid neither for usual metals, nor for materials with degenerate electron gas, because the electrons with the energy well below the Fermi level can't change their energy $E$ either through scattering or due to external fields as all these states are occupied; the Fermi distribution function leads to $f(E)=1$. Only a small part of electrons, whose energy is close to the Fermi level, are able to move randomly. The relations (1) and (2) are valid only for materials with non-degenerate electron gas.

\section{The Basic Transport Characteristics for Degenerate Materials with One Type of Randomly Moving Charge Carriers}

The effective density of randomly moving electrons $n_{\text {eff }}$ can be presented as [1]-[3]

$$
n_{\text {eff }}=\int g(E) f(E)[1-f(E)] \mathrm{d} E=k T \int g(E)\left(-\frac{\partial f(E)}{\partial E}\right) \mathrm{d} E .
$$

This relation is valid in all cases. In the case of high degree of degeneracy, the effective density of randomly moving electrons can be expressed as

$$
n_{\text {eff }}=g_{\text {eff }}\left(E_{F}\right) k T,
$$

where $g_{\text {eff }}\left(E_{F}\right)$ is the total density of states at $E=E_{F}$, and $n_{\text {eff }}$ is proportional to the temperature $T$. The density of states at the Fermi energy can be obtained from the experimental results of the electron heat capacity measurements.

For degenerate materials with one type of randomly moving charge carriers such general expressions can be presented [2] [3]: 
- for conductivity:

$$
\sigma=q n_{\mathrm{eff}} \mu_{\text {drift }}=\frac{q^{2} D}{k T} n_{\mathrm{eff}}=\frac{1}{3} q^{2} g_{\mathrm{eff}}\left(E_{F}\right) v_{F}^{2} \tau_{F},
$$

- for drift mobility (Figure 1):

$$
\mu_{\text {drift } 1}=\frac{q D}{k T}=\frac{q v_{F}^{2} \tau_{F}}{3 k T}
$$

- for Hall mobility (Figure 2):

$$
\mu_{H 1}=R_{H 1} \sigma=\frac{q \tau_{F}}{m_{0}}
$$

- for Hall coefficient:

$$
R_{H 1}=\frac{1}{q \alpha_{\varepsilon} n_{\mathrm{eff}}}=\frac{3}{2 q g\left(E_{F}\right) E_{F}}=\frac{3}{q g\left(E_{F}\right) m_{0} v_{F}^{2}} \approx \text { const }
$$

- for plasma frequency:

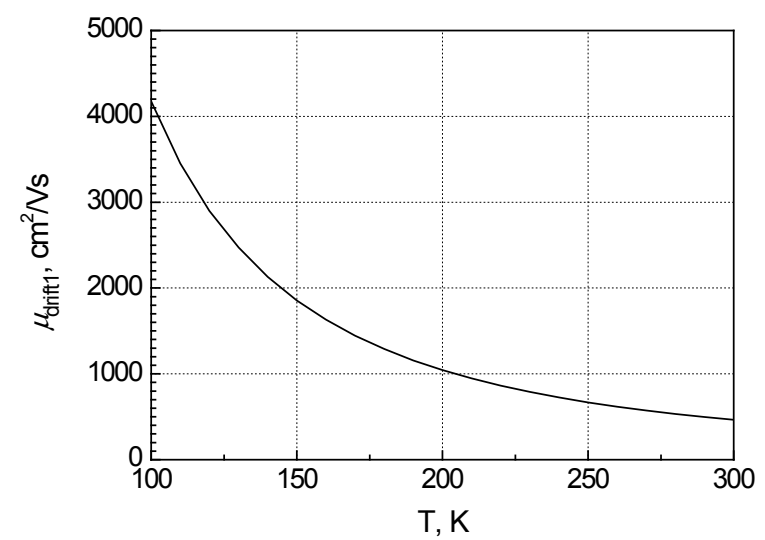

Figure 1. Drift mobility (absolute value) for single type of charge carrier dependence on temperature in $\mathrm{YBa}_{2} \mathrm{Cu}_{3} \mathrm{O}_{7}$ (calculation quantities are taken from Table 1).

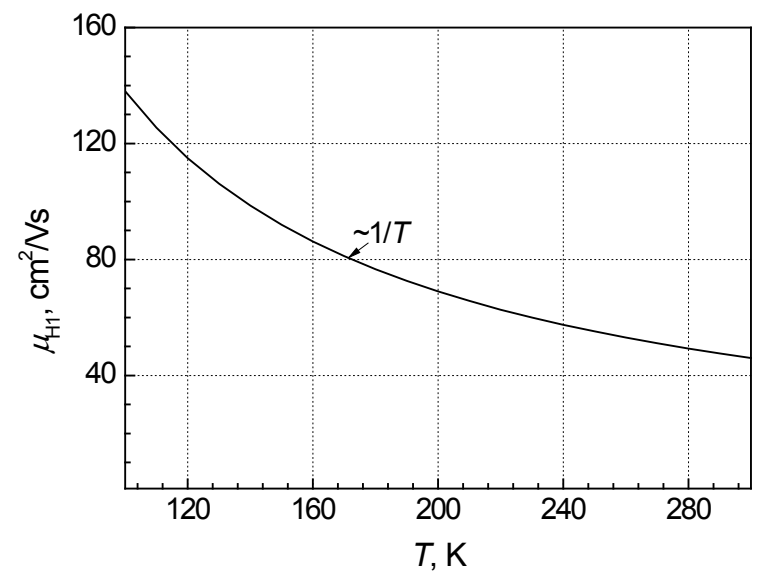

Figure 2. Hall mobility (absolute value) for single type of charge carrier dependence on temperature in $\mathrm{YBa}_{2} \mathrm{Cu}_{3} \mathrm{O}_{7}$ (calculation quantities are taken from Table 1). 
Table 1. Charge carrier transport parameters of superconductor $\mathrm{YBa}_{2} \mathrm{Cu}_{3} \mathrm{O}_{7}$ in the normal state.

\begin{tabular}{|c|c|c|}
\hline Parameter & $T=100 \mathrm{~K}$ & $T=300 \mathrm{~K}$ \\
\hline Resistivity $\rho[8]$ & $48 \mu \Omega \cdot \mathrm{cm}$ & $145 \mu \Omega \cdot \mathrm{cm}$ \\
\hline Conductivity $\sigma=1 / \rho$ & $2.08 \times 10^{4} \Omega^{-1} \cdot \mathrm{cm}^{-1}$ & $0.69 \times 10^{4} \Omega^{-1} \cdot \mathrm{cm}^{-1}$ \\
\hline Diffusion coefficient $D$ (Equation (5)) & $38.6^{*} \mathrm{~cm}^{2} / \mathrm{s}$ & $13.2^{*} \mathrm{~cm}^{2} / \mathrm{s}$ \\
\hline Drift mobility $\mu_{d r i f t 1}$ (Equation (6)) & $4176^{*}$ & $464^{*}$ \\
\hline Hall mobility $\mu_{H 1}$ (Equation (7)) & $138^{*} \mathrm{~cm}^{2} / \mathrm{V} \cdot \mathrm{s}$ & $46^{*} \mathrm{~cm}^{2} / \mathrm{V} \cdot \mathrm{s}$ \\
\hline Scattering time $\tau_{F}$ (Equation (10)) & $7.8 \times 10^{-14^{*}} \mathrm{~s}$ & $2.6 \times 10^{-14^{*}} \mathrm{~s}$ \\
\hline Effective density $n_{\text {eff }}$ of randomly moving charge carriers (Equation (4)) & $3.1 \times 10^{19} \mathrm{~cm}^{-3}$ & $9.3 \times 10^{19} \mathrm{~cm}^{-3}$ \\
\hline Free path length $l_{F}=v_{F} \tau_{F}$ & $28^{*} \mathrm{~nm}$ & $9.6^{*} \mathrm{~nm}$ \\
\hline Electron heat capacity coefficient $\gamma[9]$ & $4.5 \mathrm{~mJ} /\left(\mathrm{mol} \cdot \mathrm{K}^{2}\right)$ & \\
\hline Total density of states $g_{\text {eff }}\left(E_{F}\right)$ at Fermi surface (from $\gamma[9]$ ) & $3.63 \times 10^{21} \mathrm{eV}^{-1} \cdot \mathrm{cm}^{-3}$ & \\
\hline Fermi energy $E_{F}=m_{0} v_{F}^{2} / 2$ & $0.39 \mathrm{eV}$ & \\
\hline Plasma frequency (Equation (9)) & $1.15 \mathrm{eV}$ & \\
\hline Effective mass of randomly moving electron or hole & $m_{0}=9.1 \times 10^{-31} \mathrm{~kg}$ & \\
\hline Fermi velocity $v_{F}=\sqrt{3 D / \tau_{F}}$ & $3.85 \times 10^{7} \mathrm{~cm} / \mathrm{s}$ & \\
\hline Hall coefficient $R_{H 1}$ (Equation (8)) & $6.6 \times 10^{-3} \mathrm{~cm}^{3} / \mathrm{C}$ & \\
\hline
\end{tabular}

Note: The mark ${ }^{*}$ note that the same absolute values are for the randomly moving electrons and holes.

$$
\omega_{p}^{2}=\frac{\sigma}{\varepsilon_{0} \tau_{F}}=\frac{q^{2} g_{\text {eff }}\left(E_{F}\right) v_{F}^{2}}{3 \varepsilon_{0}} \approx \text { const ; }
$$

where $\mu_{d r i f t 1}$ is the drift mobility, and $D$ is the diffusion coefficient for single type of charge carriers; $\tau_{F}$ and $E_{F}$ is the average relaxation time and the Fermi energy of randomly moving charge carriers with velocity $v_{F}$; $R_{\mathrm{H} 1}$, and $\mu_{\mathrm{H} 1}$ are the Hall coefficient, and Hall mobility, respectively; $\varepsilon_{0}$ is the permittivity of free space; $\alpha_{\varepsilon}=E_{F} /(3 k T / 2)$. The absolute values in expressions (6)-(8) for electrons and holes are the same, but for electrons and holes they have negative or positive signs, respectively.

As shown in [6] [7], the average electron scattering time at the Fermi surface at linear resistivity dependence on temperature $T$ range in highly degenerate materials is equal:

$$
\tau_{F}=\hbar / k T,
$$

where $\hbar=h / 2 \pi$ is the Plank's constant. From Equation (8) follows that only from Hall coefficient measurement results of materials with degenerate electron gas ones could not find the total density of free electrons. From these expressions also follows that for highly degenerate materials the ratio between the drift mobility and Hall mobility is valid such relation:

$$
\frac{\mu_{d r i f t 1}}{\mu_{H 1}}=\alpha_{\varepsilon}=\frac{E_{F}}{(3 / 2) k T}=\frac{m_{0} v_{F}^{2}}{3 k T} .
$$

So, the drift mobility in highly degenerate materials can be ten or hundred times larger than Hall mobility. Equation (9) shows that plasma frequency for highly degenerate materials directly does not depend on the effective mass of charge carriers.

On the ground of both the resistivity $\rho$ [8] and the electron heat capacity coefficient $\gamma[9]$ measurement results for high quality superconductor $\mathrm{YBa}_{2} \mathrm{Cu}_{3} \mathrm{O}_{7}$ in Table 1 there are presented its charge carrier transport parameters in the normal state evaluated on the base of randomly moving charge carrier theory (near each parameter there are cited the equation according to which the mentioned value was calculated). There also has been consi- 
dered that quasiparticles electrons and holes randomly are moving independently.

It is worth here to point that the randomly moving charge carrier density $n_{\text {eff }}$ in high degenerate materials is not constant, but is proportional to temperature $T$. At linear resistivity dependence on temperature range $\tau_{F} \sim 1 / T$, and $\mu_{H 1} \sim 1 / T$, while $\mu_{d r i f t 1}^{\prime} \sim 1 / T^{2}$. Hence, one can be very careful on the interpretation of the conductivity, Hall-effect and plasma frequency measurement results.

\section{The Basic Transport Characteristics for Degenerate Materials with Two Type of Randomly Moving Charge Carriers and Its Applications to Superconductor $\mathrm{YBa}_{2} \mathrm{Cu}_{3} \mathrm{O}_{7-\delta}$}

It is pointed out that the electron-phonon scattering, not the phonon-assisted electron hopping, is highly likely responsible for in $a b$ plane transport of the high- $T_{C}$ YBCO [8]. It is considered that the linear $T$ dependence of the in-plane resistivity $\rho_{a b}(T)$ from $T_{C}$ to room temperature can be explained by the traditional electron-phonon scattering mechanism in a single-band model. In order to explain the nearly linear $T$ dependence of the Hall number $1 / q R_{H}$ from $T_{C}$ to room temperature, with magnetic field along the $c$ axis, the two-band model with positive and negative charge carriers and with particular conditions is needed.

The $a b$ plane Hall coefficient of $\mathrm{YBa}_{2} \mathrm{Cu}_{3} \mathrm{O}_{7-\delta}$ has the following characteristics [10]-[12]: a) the sign of the Hall coefficient $R_{H}$ is positive, i.e. hole-like; b) $R_{H}$ is very sensitive to the oxygen content and increases rapidly with increasing oxygen deficiency $\delta$; c) $R_{H}$ is strongly temperature dependent, that means this is a multiband system; for a well oxygenate samples $\left(T_{C}\right.$ is over $\left.90 \mathrm{~K}\right)$ the temperature dependence is such that the quantity $1 / q R_{H}$ varies linearly with $T ; \mathrm{d}$ ) in samples which are not oxygen deficient, the quantity $1 / q R_{H}$ is of the order of $10^{21} \mathrm{~cm}^{-3}$. The quantity $1 / q R_{H}$ was mistakenly accepted as the free charge carrier density, because from Equation (8) follows that for degenerate charge carriers $1 / q R_{H}=3 /\left[2 g\left(E_{F}\right) E_{F}\right]$. Moreover, for materials with degenerate electron gas and with composite electron energy band structure there is no method directly to measure the total free charge density, yet in the case of single type of charge carriers [3] (Figure 3). But the randomly moving charge carrier density can be determined by Equation (4) which really causes the transport characteristics in materials with degenerate charge carriers.

Usually in literature using a two-band model, the conductivity and the Hall coefficient are expressed in terms of the densities of both electrons $n_{e}$ and holes $n_{h}$ and their mobilities $\mu_{e}$ and $\mu_{h}$ [11] [13]-[16]:

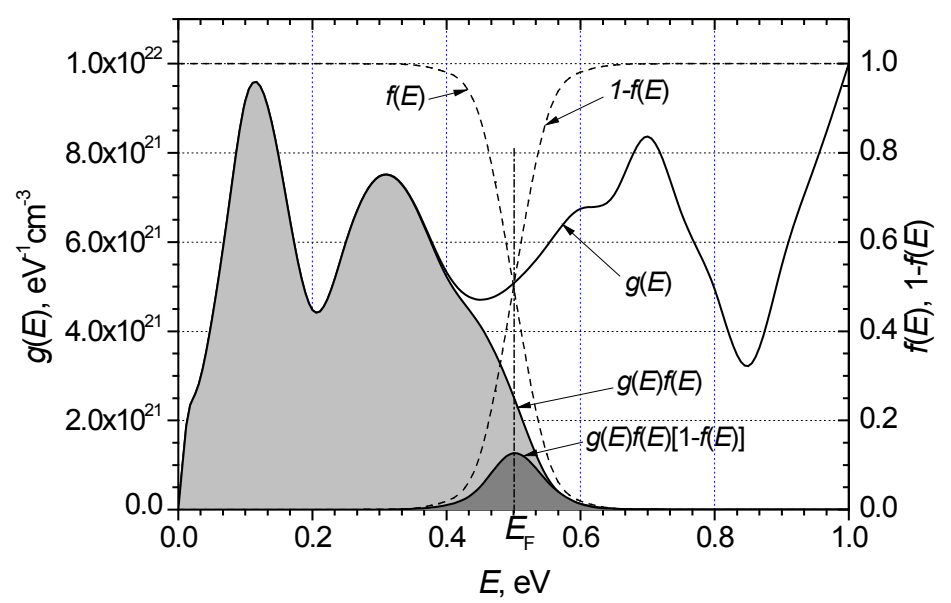

Figure 3. Illustration of density of states $g(E)$, functions $g(E) f(E)$, and $g(E) f(E)[1-f(E)]$ dependency on energy for metals and normal state superconductors with composite density of states dependency on energy. The light grey area represents the total density of electrons $n=\int g(E) f(E) \mathrm{d} E$, and the dark grey area represents the effective density of randomly moving electrons $n_{\text {eff }}$ (Equation (3)) that take part in conductivity and other kinetic processes. Additionally there are represented the Fermi functions $f(E)$ and $(1-f(E))$ dependency on energy (dashed curves, right scale). 


$$
\begin{aligned}
& \sigma=\frac{1}{\rho}=q n_{h} \mu_{h}+q n_{e} \mu_{e}, \\
& R_{H}=\frac{n_{h} \mu_{h}^{2}-n_{e} \mu_{e}^{2}}{q^{2}\left(n_{h} \mu_{h}+n_{e} \mu_{e}\right)^{2}} .
\end{aligned}
$$

In these relations on the ground that the average scattering time is defined by scattering time on the Fermi surface: $\langle\tau\rangle=\tau_{F}$, there was accepted that the drift mobility is equal to the Hall mobility, which, as it is seen from Equations (6) (Figure 1) and (7) (Figure 2), is completely incorrect assumption. In this two-band model the temperature dependence of $R_{H}$ reflects a temperature dependent compensation of Hall voltages caused by holes and electrons. It is not generally possible to uniquely determine the values $n_{e}, n_{h}, \mu_{e}$ and $\mu_{h}$ only from experimental data of conductivity and Hall effect measurements. In order to explain $R_{H} \sim 1 / T$ and $\rho \sim T$ behavior in terms of two-band model ones have to assume a very unusual special relations among the parameters. In [13], it is assumed that the effective densities of electrons and holes do not vary with temperature, but their mobilities $\mu_{h}$ and $\mu_{e}$ have the forms

$$
\begin{aligned}
& \mu_{h}^{-1}=A(1+B T), \\
& \mu_{e}^{-1}=C(1+D T),
\end{aligned}
$$

with $B \neq D$. Eight parameters are adjusted in order to give an accurate fit to combined resistivity and Hall data above $98 \mathrm{~K}$.

In [8], it is proposed that the key quantity is not $R_{H}$, but the Hall angle which at weak magnetic field $\Theta_{H} \approx E_{H} / E_{x}$ is proportional to $T^{-2}$ (here $E_{H}=E_{y}$ is the Hall electric field strength and $E_{x}$ is the applied electric field strength in the $x$ direction). It has been considered that it follows from that in a wide variety of substances and samples ones find that Hall angle

$$
\Theta_{H}=\omega_{c} \tau_{H}=\frac{1}{A+B T^{2}} .
$$

Thus, the relaxation time $\tau_{H}$ which determines $\Theta_{H}$ must be $\sim T^{-2}$ rather then $T^{-1}$. According to Andersen theory idea [8], that $\tau_{H}$ is the spinon mean free time since the orbital magnetic field part of the Hamiltonian commutates with the anomalous interaction which causes by scattering of the quasiparticles carrying the charge. From his model follows that in the resistivity measurements well above the $T_{C}$ (without the magnetic field) the scattering time changes as $1 / T$, but in the measurement of Hall effect, i.e. in the presence of magnetic field, the scattering time changes as $1 / T^{2}$. The measurement of the magnetoresistance in single-crystal $\mathrm{YBa}_{2} \mathrm{Cu}_{3} \mathrm{O}_{7-\delta}$ above $95 \mathrm{~K}$ is smaller than $1 \%$ of the resistance [17]. So, there is no real evidence that in weak magnetic fields the charge carrier scattering time drastically changes. Also one can see from Equation (7) that the same scattering time is in the conductivity $\sigma$ expression and it unambiguously gives that Hall coefficient does not depend on the scattering time. The same conclusion follows from Equation (13).

As it was mentioned earlier, Equation (13) was derived on the misconstruction base that drift and Hall mobilities for degenerate materials coincide. Let us define the Hall coefficient on the ground of the effective density of randomly moving charge carriers for two types of charge carriers: holes and electrons. The general expressions for conductivity and Hall coefficient can be presented as [18] [19]:

$$
\begin{gathered}
\sigma=\sigma_{h}+\sigma_{e}, \\
R_{H 2}=\frac{R_{H h} \sigma_{h}^{2}+R_{H e} \sigma_{e}^{2}}{\sigma^{2}}=\frac{\mu_{H h} \sigma_{h}+\mu_{H e} \sigma_{e}}{\sigma^{2}} .
\end{gathered}
$$

There the relation $R_{H h, e} \sigma_{h, e}=\mu_{H h, e}$ has been used. It is suggested that holes in $\mathrm{YBa}_{2} \mathrm{Cu}_{3} \mathrm{O}_{7-\delta}$ arise from the $\mathrm{CuO}_{2}$ planes, and are very similar to the holes in $\mathrm{La}_{2} \mathrm{CuO}_{4}$, while the carriers in the $\mathrm{Cu}-\mathrm{O}$ chains are electrons [19]. Including the effective densities of randomly moving holes and electrons, and the drift and Hall mobilities, Equations (17) and (18) can be rewritten in the following way:

$$
\sigma=q p_{\text {heff }} \mu_{h d r i f t}+q n_{\text {eeff }} \mu_{\text {edrift }},
$$




$$
R_{H 2}=\frac{q p_{\text {heff }} \mu_{\text {hdrift }} \mu_{H h}-q n_{\text {eeff }} \mu_{\text {edrift }} \mu_{H e}}{\left(q p_{\text {heff }} \mu_{h d r i f t}+q n_{\text {eeff }} \mu_{\text {edrift }}\right)^{2}} .
$$

In degenerate materials the effective densities of randomly moving charge carriers and their absolute values of drift and Hall mobilities can be presented in the following way [1]-[3]:

$$
\begin{aligned}
& n_{\text {eeff }}=g_{e}\left(E_{F}\right) k T, \\
& p_{\text {heff }}=g_{h}\left(E_{F}\right) k T, \\
& \mu_{\text {edrift }}=\mu_{h d r i f t}=\frac{q v_{F}^{2} \tau_{F}}{3 k T}, \\
& \mu_{H h}=\mu_{H e}=\frac{q \tau_{F}}{m_{0}},
\end{aligned}
$$

where $g_{e}\left(E_{F}\right)$ and $g_{h}\left(E_{F}\right)$ are electron-like and hole-like effective densities of states at the Fermi surface, respectively; $v_{F}$ and $\tau_{F}$ are the velocity and the average scattering time of charge carriers at the Fermi surface, respectively. Accounting Equations (21)-(24), Equations (19) and (20) can be presented in such a form:

$$
\begin{aligned}
& \sigma=q \frac{q v_{F}^{2} \tau_{F}}{3 k T}\left(g_{h}\left(E_{F}\right)+g_{e}\left(E_{F}\right)\right) k T=\left(\frac{1}{3}\right) q^{2} v_{F}^{2} \tau_{F} g_{e f f}\left(E_{F}\right), \\
& R_{H 2}=\frac{q^{3} v_{F}^{2} \tau_{F}^{2} g_{e f f}\left(E_{F}\right)}{3 \sigma^{2} m_{0}}\left[1-2 \cdot \frac{g_{e}\left(E_{F}\right)}{g_{e f f}\left(E_{F}\right)}\right]=\frac{3}{q v_{F}^{2} m_{0} g_{e f f}\left(E_{F}\right)}\left[1-2 \cdot \frac{g_{e}\left(E_{F}\right)}{g_{e f f}\left(E_{F}\right)}\right] .
\end{aligned}
$$

or in simplified form (by using Equation (8)):

$$
R_{H 2}=R_{H 1}\left[1-2 \cdot \frac{g_{e}\left(E_{F}\right)}{g_{\text {eff }}\left(E_{F}\right)}\right],
$$

where $g_{\text {eff }}\left(E_{F}\right)=g_{h}\left(E_{F}\right)+g_{e}\left(E_{F}\right)$ is the total density of states at $E=E_{F} \cdot R_{H 1}$ is the Hall coefficient expression for single type of randomly moving charge carriers. There it was considered that for randomly moving charge carriers (electrons and holes) the effective mass $m_{h}^{*}=m_{e}^{*}=m_{0}$. It is difficult to believe that the effective mass of randomly moving charge carriers in degenerate materials can change with temperature; the latter result would mean that the Fermi energy $E_{F}=m^{*} v_{F}^{2} / 2$ must change at the same scale.

Thus, one can present Equation (27) in such simplified form:

$$
R_{H 2}=R_{H 1}\left[1-2 \cdot \frac{g_{e}\left(E_{F}\right)}{g_{\text {eff }}\left(E_{F}\right)}\right]=\eta R_{H 1} .
$$

where

$$
\eta=\frac{R_{H 2}}{R_{H 1}}=\frac{g_{h}\left(E_{F}\right)-g_{e}\left(E_{F}\right)}{g_{\text {eff }}\left(E_{F}\right)}=1-2 \cdot \frac{g_{e}\left(E_{F}\right)}{g_{\text {eff }}\left(E_{F}\right)} .
$$

The quantity $\eta$ plays the role of compensation of voltages caused by holes and electrons in Hall effect measurement in degenerate material, and the quantity $1 / \eta$ can be named as a compensation degree factor. Considering that Hall coefficient $R_{H 1}$ for degenerate material with single charge carriers is defined by Equation (8), from the measurement of Hall coefficient $R_{H 2}$ in degenerate material with electrons and holes it possible to determine the compensation degree factor $1 / \eta$, and the part of electron-like and hole-like densities of states at the Fermi surface, and they changes with temperature.

It was interesting to find such electron-like and hole-like densities of states dependences on temperature in such a way that they could cause the Hall coefficient $R_{H 2}$ proportionality to $1 / T$, and at the same time the Hall 
mobility $\mu_{H 2}$ proportionality to $1 / T^{2}$. The obtained electron-like and hole-like densities of states dependences on temperature are presented in Figure 4. From this figure follows that parameter $1 / \eta$ is proportional to temperature $T$ (Figure 5). Thus, the slow increase of electron-like density of states and decrease of hole-like density of states with temperature in principle can produce that Hall coefficient proportionality to $1 / T$ in superconductor $\mathrm{YBa}_{2} \mathrm{Cu}_{3} \mathrm{O}_{7-\delta}$ due to compensation effect at temperatures well above the transition temperature $T_{C}$ (Figure 6).

From Equations (7), (18) and (28) the effective expression of Hall mobility for two types of randomly moving charge carriers (holes and electrons) can be presented in the following form:

$$
\mu_{H 2}=\mu_{H h} \frac{\sigma_{h}}{\sigma}-\mu_{H e} \frac{\sigma_{e}}{\sigma}=\frac{q \tau_{F}}{m_{0}}\left[1-2 \cdot \frac{g_{e}\left(E_{F}\right)}{g_{\text {eff }}\left(E_{F}\right)}\right]=\eta \mu_{H 1},
$$

where $\mu_{H 1}=q \tau_{F} / m_{0}$ is the Hall mobility for single type of randomly moving charge carriers (Figure 2). The Hall mobility $\mu_{H 2}$ dependence on temperature for randomly moving holes and electrons with the densities of states presented in Figure 4 is presented in Figure 7. The obtained Hall mobility parameter $\mu_{H 2}$ (Figure 7) dependence on temperature explains the proportionality to $1 / T^{2}$ of the experimental results [20].

In the case of randomly moving electron and hole quasiparticles, the expressions for Hall coefficient (Equation (28)) and for Hall mobility (Equation (30)), and considering that conductivity dependence on temperature

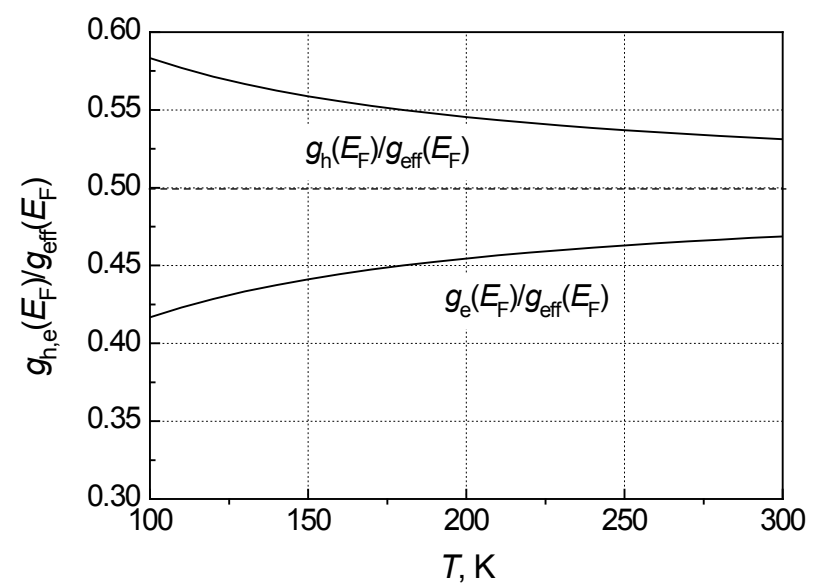

Figure 4. The hole-like and electron-like densities of states dependence on temperature for superconductor $\mathrm{YBa}_{2} \mathrm{Cu}_{3} \mathrm{O}_{7-\delta}$, which cause the Hall coefficient $R_{H 2} \sim 1 / T$.

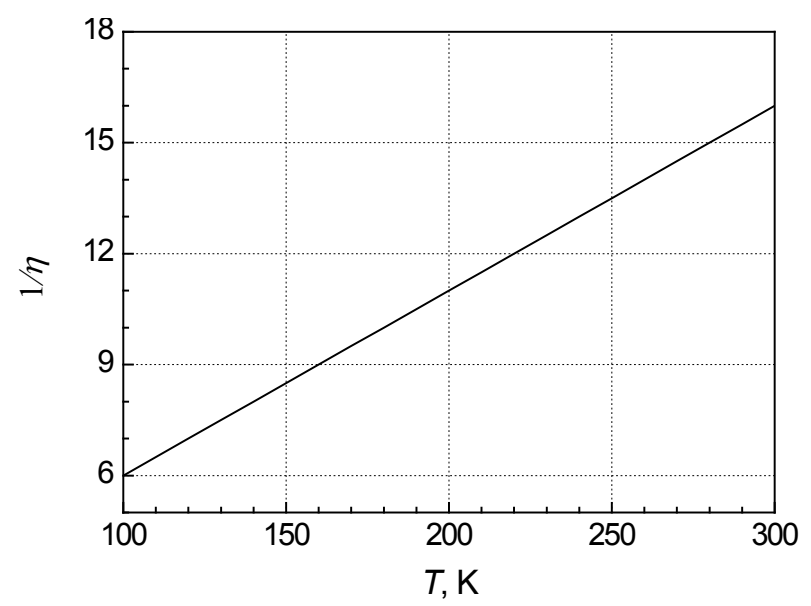

Figure 5. The compensation degree factor dependence on temperature for superconductor $\mathrm{YBa}_{2} \mathrm{Cu}_{3} \mathrm{O}_{7-\delta}$. 


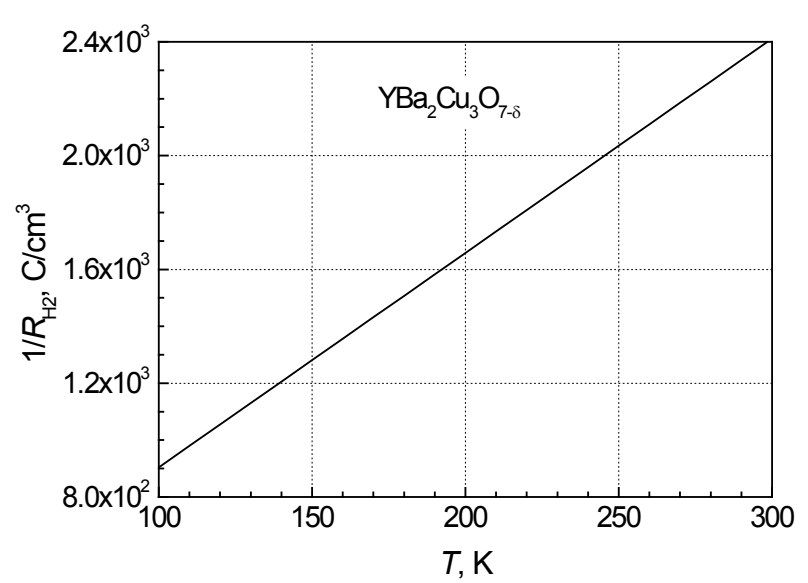

Figure 6. Hall coefficient $1 / R_{H 2}$ dependence on temperature in the normal state temperature range for $\mathrm{YBa}_{2} \mathrm{Cu}_{3} \mathrm{O}_{7-\delta}$.

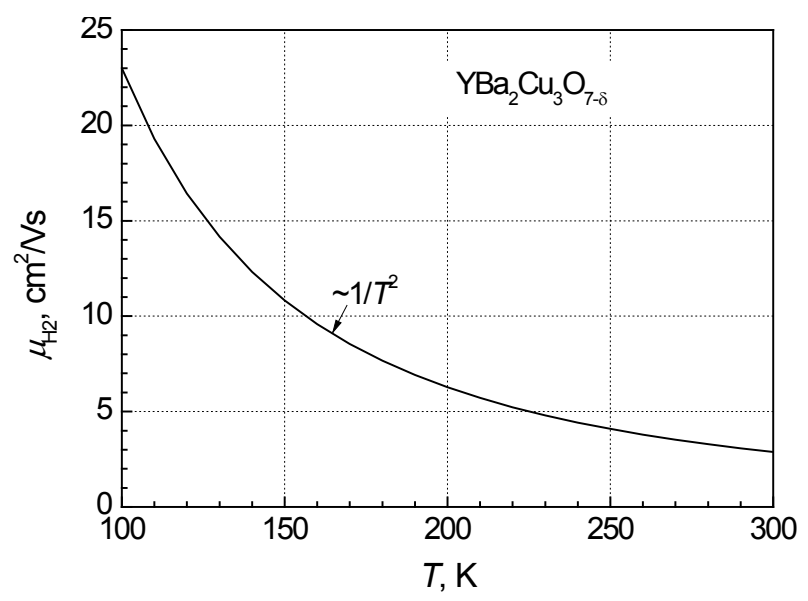

Figure 7. The Hall mobility $\mu_{H 2}$ value dependence on temperature for randomly moving holes and electrons in $\mathrm{YBa}_{2} \mathrm{Cu}_{3} \mathrm{O}_{7-\delta}$ with the densities of states presented in Figure 4.

are only due to $\tau_{F} \sim 1 / T$, depend only on the ratio of electron-like density of states to the total density of states $g_{\text {eff }}\left(E_{F}\right)$, i.e. depend only on parameter $1 / \eta$.

As pointed in [5], that near optimum doping rising the temperature has a similar effect, possibly by releasing carriers from localized states or by increasing the effective hole doping in the $\mathrm{CuO}_{2}$ layers, or by changing the effective band structure, i.e. changing the effective density of electron-like and hole-like states. In [21], it is pointed that due to displacement of oxygen atoms in chains there can be changes of density of states in-plane and in chain states.

So, when the hole-like and electron-like densities of states are close to one other then the small changes of these densities with temperature can produce the drastic changes in temperature dependence of Hall coefficient $R_{H 2}$ and Hall mobility $\mu_{H 2}$ in the case of two types of charge carriers: electrons and holes.

\section{Conclusion}

This work tries to attract attention to interpretation of transport properties of superconductor $\mathrm{YBa}_{2} \mathrm{Cu}_{3} \mathrm{O}_{7-\delta}$ in temperature range well over the transition temperature $T_{C}$. On the base of the density of randomly moving charge carriers and their drift and Hall mobilities, the general expressions for Hall coefficient and Hall mobility for two types of charge carriers - holes and electrons were derived. Considering that Hall coefficient $R_{H 1}$ for degenerate material with single type of charge carriers is completely defined, from the measurement of Hall coefficient $R_{H 2}$ in degenerate material with electrons and holes it possible to determine the compensation degree 
factor $1 / \eta$, and the part of electron-like and hole-like densities of states at the Fermi surface, and they changes with temperature. When the hole-like and electron-like densities of states are close to other ones, the small changes of these densities with temperature can produce the drastic changes in temperature of Hall coefficient $R_{H 2}$ and Hall mobility $\mu_{H 2}$. It is shown that scattering time $\tau_{F}$ always has its usual dependence on temperature: $\tau_{F}$ $\sim 1 / T$.

\section{Acknowledgements}

This study was partially funded by European Social Fund under Global Grant measure No. VP1-3.1-ŠMM-0703-040.

\section{References}

[1] Palenskis, V. (2013) Drift Mobility, Diffusion Coefficient of Randomly Moving Charge Carriers in Metals and Other Materials with Degenerate Electron Gas. World Journal of Condensed. Matter Physics, 3, 73-81. http://dx.doi:104236/wjcmp.2013.31013

[2] Palenskis, V. (2014) The Effective Density of Randomly Moving Electrons and Related Characteristics of Materials with Degenerate Electron Gas. AIP Advances, 4, 047119-1(9). http://dx.doi.org/10.1063/1.4871757

[3] Palenskis, V. (2013) A Novel View to Free Electron Theory. Materials with Degenerate Electron Gas. LAP LAMBERT Academic Publishing, Saarbrücken.

[4] Friedman, T.A., Rabin, M.W., Giapintzakis, J., Rice, J.P. and Ginsberg, D.M. (1990) Direct Measurement of the Anisotropy of the Resistivity in the $a-b$ Plane of Twin-Free, Single-Crystal, Superconducting $\mathrm{YBa}_{2} \mathrm{Cu}_{3} \mathrm{O}_{7-\delta}$. Physical Review B, 42, 6217-6221. http://dx.doi.org/10.1103/PhysRevB.42.6217

[5] Waldram, J.R. (1996) Superconductivity of Metals and Cuprates, Chapter 14: Normal-State Transport Properties in the Cuprates. Institute of Physics Publishing, Ltd., London.

[6] Devillers, M.A.C. (1984) Lifetime of Electrons in Metals at Room Temperature. Solid State Communications, 49 , 1019-1022. http://dx.doi.org/10.1016/0038-1098(84)90413-7

[7] Lemberger, T.R. and Coffey, L. (1988) Effects of Inelastic Electron Scattering on Properties of High-Temperature Superconductors. Physical Review B, 38, 7058-7060. http://dx.doi.org/10.1103/PhysRevB.38.7058

[8] Andersen, P.W. (1997) The Theory of Superconductivity in the High- $T_{C}$ Cuprates. Chapter 3. Normal State Properties in the High- $T_{C}$ Superconductors: Evidence for Non-Fermi Liquid States. Princeton Series in Physics, Princeton University Press, Princeton.

[9] Volovik, G.E. (1989) Nonzero State Density in Superconductors with High Transition Temperature. Journal of Experimental and Theoretical Physics Letters, 49, 790-792.

[10] Xing, D.Y., Liu, M. and Ting, C.S. (1988) Out-off-Plane Transport Mechanism in the High- $T_{C}$ Oxide Y-Ba-Cu-O. Physical Review B, 38, 11992-11995. http://dx.doi.org/10.1103/PhysRevB.38.11992

[11] Dresselhaus, M.S. (2001) Solid State Physics, Part IV: Superconducting Properties of Solids. 6.732, Fall.

[12] Lu, Y., Zhao, B.R., Yuan, C.W., Zhang, Y.Z., Wang, H.S., Shi, Y.H., Xu, P. and Li, L. (1989) The Hall Coefficient of $\mathrm{YBa}_{2} \mathrm{Cu}_{3} \mathrm{O}_{7-\mathrm{x}}$ Thin Films with (100) Orientation. Zeitschrift für Physik B Condensed Matter, 74, 283-287. http://dx.doi.org/10.1007/BF01307874

[13] Eagles, D.M. (1989) Concentrations and Mobilities of Holes and Electrons in a Crystal of a $90 K$ Oxide Superconductor from Analysis of ab-Plane Resistivity and Hall Data. Solid State Communications, 69, 229-234. http://dx.doi.org/10.1016/0038-1098(89)90840-5

[14] Xing, D.Y., Liu, M. and Ting, C.S. (1988) Out-off-Plane Transport Mechanism in the High- $T_{C}$ Oxide Y-Ba-Cu-O. Physical Review B, 38, 11992-11995. http://dx.doi.org/10.1103/PhysRevB.38.11992

[15] Xing, D.Y. and Ting, C.S. (1988) Two-Band Model for Anisotropic Hall Effect in High- $T_{C}$ Y-Ba-Cu-O. Physical Review B, 38, 5134-5137. http://dx.doi.org/10.1103/PhysRevB.38.5134

[16] Ginsberg, D.M. (Ed.) (1992) Physical Properties of High Temperature Superconductors. World Scientific Publishing Co., Singapure.

[17] Hikita, M. and Suzuki, M. (1989) Magnetoresistance and Thermodynamic Fluctuations in Single- $\mathrm{Crystal} \mathrm{YBa}_{2} \mathrm{Cu}_{3} \mathrm{O}_{\mathrm{y}}$. Physical Review B, 39, 4756-4759. http://dx.doi.org/10.1103/PhysRevB.39.4756

[18] Ziman, J.M. (1972) Principles of the Theory of Solids. Cambridge University Press, Cambridge. http://dx.doi.org/10.1017/CBO9781139644075

[19] Markiewicz, R.S. (1988) Simple Model for the Hall Effect in $\mathrm{YBa}_{2} \mathrm{Cu}_{3} \mathrm{O}_{7-\delta}$. Physical Review B, 38, 5010-5011. 
$\underline{\text { http://dx.doi.org/10.1103/PhysRevB.38.5010 }}$

[20] Foro, L., Raki, M., Henry, J.Y. and Ayache, C. (1989) Hall Effect and Thermoelectric Power of an $\mathrm{YBa}_{2} \mathrm{Cu}_{3} \mathrm{O}_{6.8} \mathrm{Single}$ Crystal. Solid State Communications, 69, 1097-1101. http://dx.doi.org/10.1016/0038-1098(89)90493-6

[21] Anisimov, V.I., Korotin, M.A. and Afanasyev, I.V. (1988) Influence of Displacement of Chain Oxygen Atoms on the Electronic Structure of $\mathrm{YBa}_{2} \mathrm{Cu}_{3} \mathrm{O}_{7}$. The Physics of Metals and Metallography, 66, 161-193. 